\title{
1 The chromosome-scale genome assembly of Jasminum sambac var. unifoliatum provide insights into the formation 3 of floral fragrance
}

4 Chengzhe Zhou ${ }^{1,2,3}$, Chen $\mathrm{Zhu}^{1,2,3}$, Caiyun Tian ${ }^{1,3}$, Siyi Xie ${ }^{1,3}$, Kai $\mathrm{Xu}^{1,3}$, Linjie 5 Huang ${ }^{1,3}$, Shengjing Wen ${ }^{1,3}$, Cheng Zhang ${ }^{1,3}$, Zhongxiong Lai ${ }^{1,2}$, Yuling Lin ${ }^{1,2, *}$,

6 Yuqiong Guo ${ }^{1,3, *}$

$7 \quad{ }^{*}$ Corresponding author

$8{ }^{1}$ College of Horticulture, Fujian Agriculture and Forestry University, Fuzhou, 350002, 9 China ${ }^{2}$ Institute of Horticultural Biotechnology, Fujian Agriculture and Forestry University, Fuzhou, 350002, China

${ }^{3}$ Tea Industry Research Institute, Fujian Agriculture and Forestry University, Fuzhou, 350002, China

\section{Abstract}

The high-quality genome assembly of the JSU-FSP provides valuable information on how jasmine produces the abundant VTs and VPBs that synergistically contribute to the pleasant aroma of jasmine flower.

\section{Introduction}

Jasmine (Jasminum sambac) is a tropical or subtropical plant that is notable for the sweet, heady fragrance of its flowers. The high accumulation of volatile terpenes (VTs) and volatile phenylpropanoid/benzenoids (VPBs) in jasmine flowers creates a natural source of essential oil to meet consumer demand all over the world. Additionally, jasmine is also used to produce scented tea, which is reprocessed from finished tea by absorbing aroma volatiles of the fresh flowers and has been favoured since antiquity [1]. Cultivar jasmine varieties mainly belong to three major groups: J. sambac var. unifoliatum (JSU, single-petal type), J. sambac var. bifoliatum (JSB, double-petal type), and J. sambac var. trifoliatum (JST, multi-petal type). The flowers of JSU, with the best aroma quality, are considered as the optimal materials for the production of 
high-grade jasmine tea [2]. However, the lack of a reference genome limits the studies of basic and applied biology on the jasmine. The genetic bases for the rich production of VTs and VPBs remain largely unclear. While recently the reference genomes of JSB [3] and JST [4] cultivars were de novo sequenced and assembled, no JSU genome has yet been reported.

\section{Results and discussions}

Here, we assembled a reference genome for the autotriploid JSU cultivar 'Fuzhou single-petal' (JSU-FSP). According to the 21-mer analysis of $45.14 \mathrm{~Gb}$ Illumina reads and flow cytometry, we estimated that the haploid genome size of JSU-FSP is $\sim 471.23 \mathrm{Mb}$ and $\sim 498.35 \mathrm{Mb}$, respectively. We initially assembled the genome by hifiasm v0.16 with $85.89 \mathrm{~Gb}$ PacBio HiFi reads, resulting in a $495.60 \mathrm{Mb}$ sequence assembly with a contig N50 of $16.88 \mathrm{Mb}$. The BUSCO and CEGMA analyses showed that the completeness of the JSU-FSP genome was $97.83 \%$ and $98.91 \%$, respectively, which indicated that the excellent continuity and completeness of the assembly. Then, all the contigs were corrected and orientated by $\mathrm{Hi}-\mathrm{C}$ onto 13 pseudo-chromosomes, generating chromosome-scale sequences of $476.92 \mathrm{Mb}$ (Fig. 1A and 1B).

The annotation results showed that there were $264.98 \mathrm{Mb}$ repetitive sequences in JSU-FSP genome, of which long terminal repeat-retrotransposons (LTR-RTs) accounted for $66.33 \%(175.77 \mathrm{Mb})$. The Ty3-gypsy were the predominant LTR-RTs (58.92 Mb), followed by Ty1-copia (36.68 Mb). We identified 30,989 protein-coding genes through integrated ab initio modeling, homology-based searches, and transcriptome analysis (Fig. 1A). The BUSCO analysis showed that $96.16 \%$ of the core plant genes were present in identified genes, indicating an excellent completeness of genes prediction.

Through comparative genomics analysis between the JSU-FSP genome and the genomes of other 11 representative plant species, we observed that JSU-FSP genome went through an ancient WGD event by estimating $K s$ distributions (Fig. 1C). The phylogenetic tree based on 448 single-copy orthologous genes illustrated that $J$. sambac diverged from the ancestors of other four species of Oleaceae plants about 
54 58 million years ago (Fig. 1D). Gene family analysis revealed that 26,638 genes in JSU-FSP were clustered into 17,446 families (Fig. 1E). The five Oleaceae plants shared 11,333 common gene families, while 1,221 gene families were unique in the JSU-FSP genome (Fig. 1F). The unique genes of JSU-FSP were clustered into "Terpenoid backbone biosynthesis" and "Sesquiterpenoid and triterpenoid biosynthesis" of the KEGG enrichment pathways, endowing jasmine with the genetic basis of heady fragrance (Fig. 1G).

As aroma volatiles of jasmine are synthesized and emitted in the opening process [5], flower buds or flowers at five different stages were selected for transcriptome sequencing (Fig. 1H). The expression of key genes was investigated to unveil the mechanism of VTs and VPBs biosynthesis. We identified that among the 73 structural genes involved in VTs and VPBs metabolic pathways, the expression of 43 genes in opening flowers was significantly higher than that in buds. This indicated the differential expression of these related genes in opening flowers was responsible for the aroma formation. In addition to de novo synthesis, plant volatiles are present in glycosidically bound forms and can be hydrolyzed to free volatiles by GH1, GH3, and GH5 families of $\beta$-glucosidase (BGLU) [6]. Phylogenetic analysis reveled that a total of 81 BGLU genes in JSU-FSP genome were clustered into GH1 (47), GH3 (20), and GH5 (14) subfamilies (Fig. 1J). Among which, 11 BGLU genes were highly expressed in opening flowers, implying that jasmine aroma is also affected by the expression of BGLU genes [7].

\section{Acknowledgements}

This work was supported by the Construction of Plateau Discipline of Fujian Province $(102 / 71201801101)$ and the Construction Project for Technological Innovation and Service System of Tea Industry Chain of Fujian Agriculture and Forestry University (K1520005A01). We thank Xin Huang from the School of Foreign Languages of Fuzhou University for her linguistic assistance; Ying $\mathrm{Yu}$ from Biomarker Technologies for assistance with sequencing; Prof. Yong Li from Henan Agricultural University for providing the genomic data of Forsythia suspensa; and Tianlong Fu 
91 from Fujian Chunlun Group Co. LTD for helping with material collection.

92

93 Data available statement

94 Raw sequencing data have been submitted to the NCBI under project number 95 PRJNA795621.

96

\section{Conflict of interests}

98 The authors declare that they have no known conflicts of interest.

A

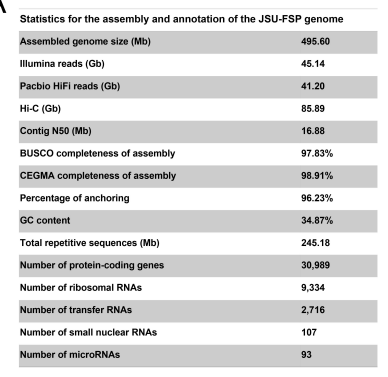

D

G

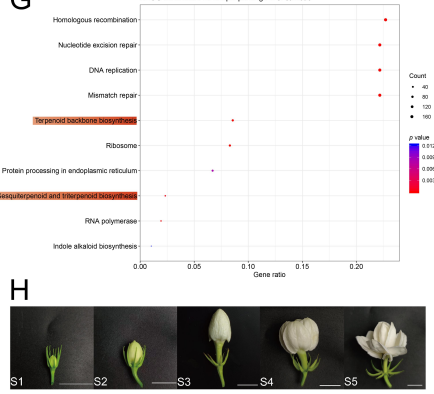

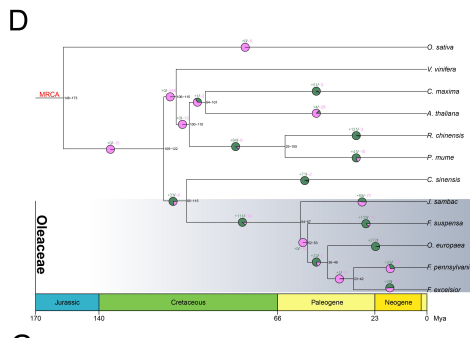

B

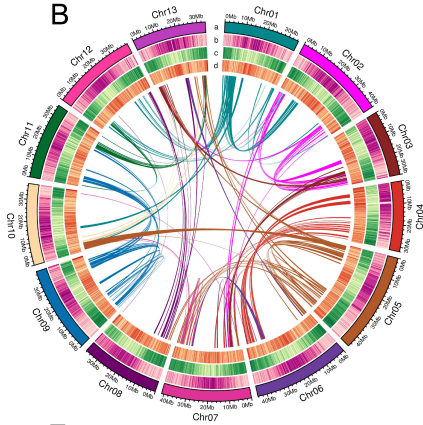

E
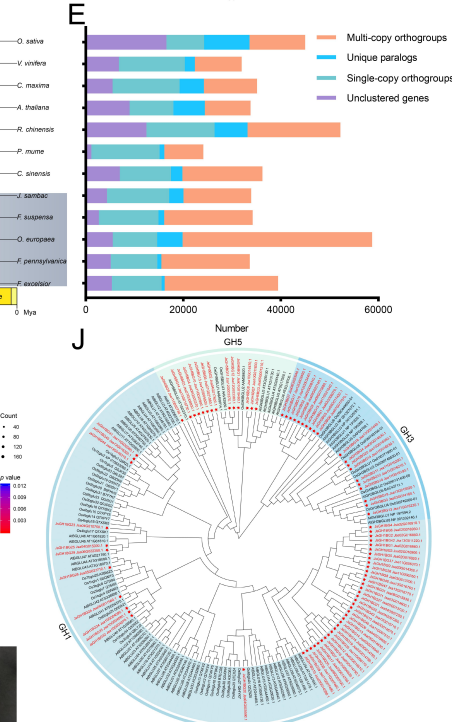

।

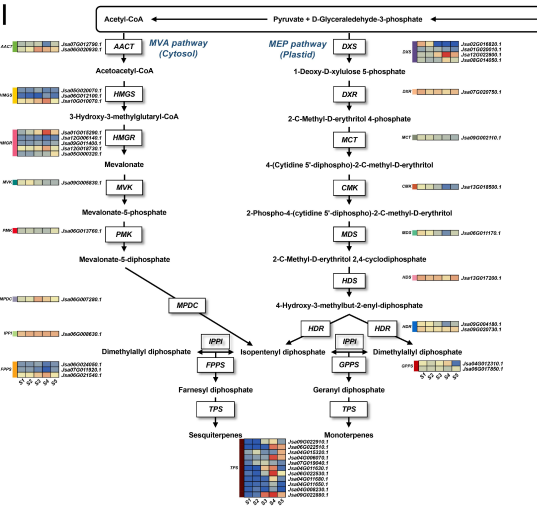

C

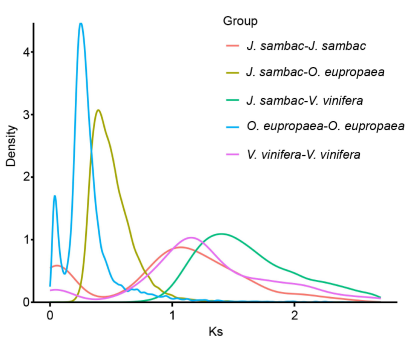

$\mathrm{F}$

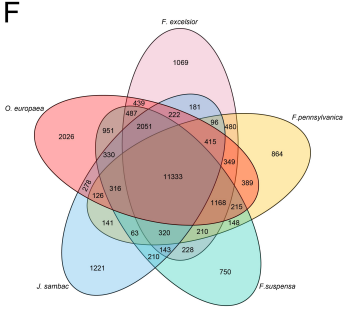

K

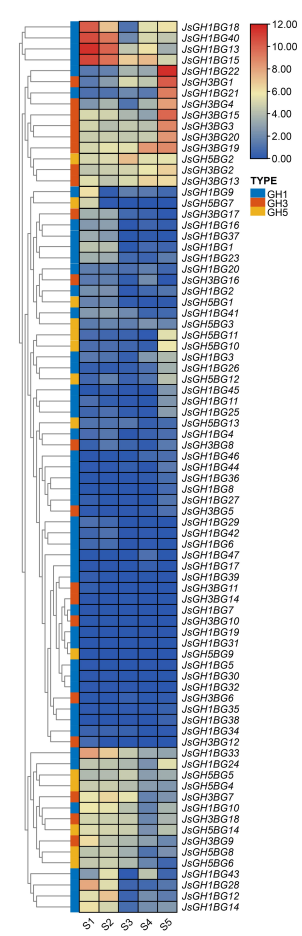


100 Figure 1. Landscape of the genome of J. sambac var. unifoliatum cv. Fuzhou single-petal (JSU-FSP). A, statistics for the assembly and annotation of the JSU-FSP genome. B, the circulars map shows chromosome ideograms (a), transposable element density (b), gene density (c), GC content (d), and syntenic blocks ( colored lines in the center). C, density distribution of $K s$ within $J$. sambac, O. europaea, and $V$. vinifera. D, phylogenetic tree with estimated divergence time and gene family expansion/contraction. E, statistics for the gene family. F, venn diagram of orthogroups within five Oleaceae plants. G, KEGG enrichment analysis for the unique paralogs in JSU-FSP genome. $\mathrm{H}$, images of five flowering stages of JSU-FSP, bar = 1 cm. I, differentially expressed genes involved in terpenes and phenylpropanoid/benzenoids metabolic pathways. J, phylogenetic tree of $\beta$-glucosidase (BGLU) proteins from JSU-FSP, A. thaliana, and O. sativa. K, the expression profile of BGLU genes in different flowering stages of JSU-FSP.

\section{References}

1. Zhou HC, Hou ZW, Wang DX et al. Large scale preparation, stress analysis, and storage of headspace volatile condensates from Jasminum sambac flowers. Food Chemistry. 2019; 286: 170-178.

2. Deng YM, Sun XB, Gu CS et al. Identification of pre-fertilization reproductive barriers and the underlying cytological mechanism in crosses among three petal-types of Jasminum sambac and their relevance to phylogenetic relationships. PLOS ONE. 2017; 12: e0176026.

3. Chen G, Mostafa S, Lu Z et al. The jasmine (Jasminum sambac) genome and flower fragrances. bioRxiv. 2021.

4. Xu SX, Ding YL, Sun JT et al. A high-quality genome assembly of Jasminum sambac provides insight into floral trait formation and Oleaceae genome evolution. Molecular Ecology Resources. 2021; 00: 1-16.

5. Zhou CZ, Zhu C, Tian CY et al. Integrated volatile metabolome, multi-flux full-length sequencing, and transcriptome analyses provide insights into the aroma formation of postharvest jasmine (Jasminum sambac) during flowering. Postharvest Biology and Technology. 2022; 183: 111726.

6. Zhou Y, Zeng LT, Gui JD et al. Functional characterizations of $\beta$-glucosidases involved in aroma compound formation in tea (Camellia sinensis). Food Research International. 2017; 96: 206-214.

7. Moon J, Watanabe N, Sakata K et al. Linalyl $\beta$-d-glucopyranoside and its 6'-O-malonate as aroma precursors from Jasminum sambac. Phytochemistry. 1994; 36: 1435-1437. 